\title{
MAXIMAL DETERMINANTS IN COMBINATORIAL INVESTIGATIONS
}

\author{
H. J. RYSER
}

1. Introduction. Let $Q$ be a matrix of order $v$, all of whose entries are 0's and 1's. Let the total number of 1's in $Q$ be $t$, and let the absolute value of the determinant of $Q$ be denoted by $|\operatorname{det} Q|$. In this paper we study the problem of determining the maximum of $|\operatorname{det} Q|$ for fixed $t$ and $v$. It turns out that this problem is closely related to the $v, k, \lambda$ problem, which has been extensively studied of late.

A $v, k, \lambda$ configuration is defined as an arrangement of $v$ elements $x_{1}, x_{2}$, $\ldots, x_{v}$ into $v$ sets $S_{1}, S_{2}, \ldots, S_{v}$ such that each set contains exactly $k$ distinct elements and such that each pair of sets has exactly $\lambda$ elements in common $(0<\lambda<k<v)$. If element $x_{j}$ belongs to set $S_{i}$, let $a_{i j}=1$; and if $x_{j}$ does not belong to $S_{i}$, let $a_{i j}=0$. The $v$ by $v$ matrix $A=\left[a_{i j}\right]$ is called the incidence matrix of the $v, k, \lambda$ configuration. These matrices have been very useful in establishing the nonexistence of certain configurations $(1 ; 2)$. A general survey of the literature pertaining to $v, k, \lambda$ configurations may be found in (4). In particular one proves that in a $v, k, \lambda$ configuration,

$$
k-\lambda=k^{2}-\lambda v
$$

and

$$
A A^{\mathrm{T}}=A^{\mathrm{T}} A=B .
$$

Here $A^{\mathrm{T}}$ denotes the transpose of the incidence matrix $A$, and the matrix $B$ has $k$ in the main diagonal and $\lambda$ in all other positions. It is easy to see that $\operatorname{det} B=k^{2}(k-\lambda)^{0-1}$, whence it follows that

$$
|\operatorname{det} A|=k(k-\lambda)^{\frac{1}{2}(n-1)} \text {. }
$$

\section{Theorems on maximal determinants.}

THEOREM 1. Let $Q$ be a 0, 1 matrix of order $v$, containing exactly $t$ 1's. Let $k$ denote a positive real, and set $\lambda=k(k-1) /(v-1)$. If $t \leqslant k v$ and $0<\lambda$ $\leqslant k-\lambda$, or if $t \geqslant k v$ and $0<k-\lambda \leqslant \lambda$, then

$$
|\operatorname{det} Q| \leqslant k(k-\lambda)^{\frac{1}{2}(0-1)} \text {. }
$$

Let $E$ be a 0,1 matrix. Let $E(x, y)$ denote the matrix formed from $E$ by replacing each 1 of $E$ by $x$ and each 0 of $E$ by $y$, where $x$ and $y$ are indeterminates. Using this notation, we may write

$$
Q_{1}=Q(-(k-\lambda) / \lambda, 1)
$$

Received May 31, 1955. 
Now set $p=(k-\lambda) / \lambda$, and define the matrix $\bar{Q}$ of order $v+1$ by

$$
\bar{Q}=\left[\begin{array}{ll}
p & z \\
z^{\mathrm{T}} & Q_{1}
\end{array}\right],
$$

where $z=(\sqrt{ } p, \ldots, \sqrt{ } p)$. By the Hadamard determinant theorem,

$$
|\operatorname{det} \bar{Q}| \leqslant \sqrt{p^{2}+v p} \prod_{i=1}^{0} \sqrt{p+s_{i}} \text {, }
$$

where $s_{i}$ denotes the sum of the squares of the $i$ th row of $Q_{1}$. Now

$$
p^{2}+v p=p\left(\frac{k-\lambda+\lambda v}{\lambda}\right)=\frac{k^{2}}{\lambda^{2}}(k-\lambda) \text {. }
$$

Moreover,

$$
s_{1}+\ldots+s_{v}=t p^{2}+\left(v^{2}-t\right)=t\left(p^{2}-1\right)+v^{2} .
$$

By hypothesis, $t \leqslant k v$ and $p^{2} \geqslant 1$, or $t \geqslant k v$ and $p^{2} \leqslant 1$. Hence we may conclude

$$
s_{1}+\ldots+s_{v} \leqslant k v\left(p^{2}-1\right)+v^{2} .
$$

Now introduce quantities $\bar{s}_{i}$ such that

$$
\bar{s}_{i} \geqslant s_{i}
$$

and

$$
\bar{s}_{1}+\ldots+\bar{s}_{v}=v\left(k p^{2}+v-k\right) .
$$

By (3),

$$
\begin{aligned}
\sum_{i=1}^{b}\left(p+\bar{s}_{i}\right) & =v\left(k p^{2}+v-k+p\right)=v\left[k p^{2}+(\lambda v-\lambda k+k-\lambda) / \lambda\right] \\
& =v k p(p+1)=v(k-\lambda) k^{2} / \lambda^{2} .
\end{aligned}
$$

Since the geometric mean of $v$ positive quantities is less than or equal to their arithmetic mean, we may write

$$
\prod_{i=1}^{v}\left(p+\bar{s}_{i}\right) \leqslant\left(\frac{1}{v} \sum_{i=1}^{v}\left(p+\bar{s}_{i}\right)\right)^{v},
$$

whence

$$
\prod_{i=1}^{v}\left(p+\bar{s}_{i}\right) \leqslant(k-\lambda)^{v} k^{2 v} / \lambda^{2 v}
$$

Hence by (2),

(6) $|\operatorname{det} \bar{Q}| \leqslant \frac{k}{\lambda} \sqrt{k-\lambda} \prod_{i=1}^{v} \sqrt{p+\bar{s}_{i}}$

$$
\leqslant \frac{k}{\lambda} \sqrt{k-\lambda}\left(\frac{k}{\lambda} \sqrt{k-\lambda}\right)^{v}=\left(\frac{k}{\lambda} \sqrt{k-\lambda}\right)^{o+1} \text {. }
$$


To evaluate $\operatorname{det} \bar{Q}$, multiply row one by $-1 / \sqrt{ } p$ and add the resulting row to each of the other rows. From (6) it follows that

$$
|\operatorname{det} \bar{Q}|=p|\operatorname{det} Q(-k / \lambda, 0)| \leqslant(k \sqrt{k-\lambda} / \lambda)^{0+1} \text {. }
$$

But

$$
\begin{gathered}
|\operatorname{det} Q(-k / \lambda, 0)|=(k / \lambda)^{0}|\operatorname{det} Q|, \text { whence } \\
p|\operatorname{det} Q| \leqslant \frac{k}{\lambda}(\sqrt{k-\lambda})^{0+1},
\end{gathered}
$$

and

$$
|\operatorname{det} Q| \leqslant k(\sqrt{k-\lambda})^{\circ-1} .
$$

Using the notation of Theorem 1 , we have

THEOREM 2. If $|\operatorname{det} Q|=k(k-\lambda)^{\frac{1}{2}(0-1)}$, then $Q$ is the incidence matrix of a $v, k, \lambda$ configuration.

If equality holds in Theorem 1 , then

$$
p\left|\operatorname{det} Q\left(-\frac{k}{\lambda}, 0\right)\right|=\left(\frac{k \sqrt{k-\lambda}}{\lambda}\right)^{0+1} \text {, }
$$

and by (7),

$$
|\operatorname{det} \bar{Q}|=(k \sqrt{k-\lambda} / \lambda)^{0+1} .
$$

Equality in (6) implies equality in (5) and (4). But for equality to hold in (4), we must have

$$
p+\bar{s}_{i}=(k-\lambda) k^{2} / \lambda^{2} .
$$

But then the equality in (6) implies

$$
\bar{Q} \bar{Q}^{\mathbf{T}}=\frac{k^{2}(k-\lambda)}{\lambda^{2}} I,
$$

where $I$ is the identity matrix of order $v+1$. Thus

$$
Q_{1} Q_{1}^{\mathbf{T}}=\frac{k^{2}}{\lambda^{2}}(k-\lambda) I-p S,
$$

where $Q_{1}=Q(-p, 1)$, and $S$ is the $v$ by $v$ matrix of all 1 's. Let $e$ denote the number of 1 's in row $r$ of $Q$. Then

$$
p^{2} e+(v-e) \cdot 1=\frac{k^{2}}{\lambda^{2}}(k-\lambda)-p,
$$

and

$$
\left(p^{2}-1\right) e=\frac{k^{2}}{\lambda^{2}}(k-\lambda)-p-v,
$$

whence we conclude that $e=k$. Let $f$ denote the inner product of rows $r$ and $s$ of $Q$, where $r \neq s$. Then 


$$
f p^{2}-2(k-f) p+v-2 k+f=-p
$$

whence

$$
f\left(p^{2}+2 p+1\right)=2 k p-p+2 k-v
$$

and $f k^{2} / \lambda^{2}=k^{2} / \lambda$. Thus $f=\lambda$, and $Q$ is the incidence matrix of a $v, k, \lambda$ configuration.

It is now clear that we have established the following:

Theorem 3. Let $Q$ be a 0, 1 matrix of order $v$, containing exactly $t$ 1's. Let $k=t / v$ and set $\lambda=k(k-1) /(v-1)$, with $0<\lambda<k<v$. Then

$$
|\operatorname{det} Q| \leqslant k(k-\lambda)^{\frac{1}{2}(0-1)} \text {, }
$$

and equality holds if and only if $Q$ is the incidence matrix of $a v, k, \lambda$ configuration.

Consider once again Theorem 1 . Note that $(k-\lambda) / \lambda=(v-k) /(k-1)$. Thus the requirement $\lambda \leqslant k-\lambda$ means $k \leqslant \frac{1}{2}(v+1)$, and $k-\lambda \leqslant \lambda$ means $k \geqslant \frac{1}{2}(v+1)$. Suppose that $k=\frac{1}{2}(v+1)$. Then if $Q$ is a 0,1 matrix with no restriction on the number of 1 's, we must have

$$
|\operatorname{det} Q| \leqslant \frac{(v+1)^{\frac{1}{2(v+1)}}}{2^{v}} .
$$

The incidence matrix associated with the case of equality has parameters $v=4 \lambda-1, k=2 \lambda, \lambda=\lambda$. These incidence matrices give rise to the Hadamard matrices of order $4 \lambda$ (3). The determination of the maximum of $|\operatorname{det} Q|$, where $Q$ is of arbitrary order $v$, is an unsolved problem of considerable difficulty (5).

If we place no restriction on the number of 1 's in the 0,1 matrix $Q$ of order $v$ and assume that $|\operatorname{det} Q|=k(k-\lambda)^{\frac{1}{2}(0-1)}$, then we may not conclude in general that $Q$ is the incidence matrix of a $v, k, \lambda$ configuration. For example, let $A$ be an incidence matrix of a $v, k, \lambda$ configuration with $v-2 k>0$. Define its complement $C$ by $A+C=S$, where $S$ is the matrix of all 1's. The complement of $A$ is again a $v, k, \lambda$ configuration with parameters $\bar{v}=v, \bar{k}=v-k$, and $\bar{\lambda}=v-2 k+\lambda$. Note that

$$
|\operatorname{det} C|=(v-k)(k-\lambda)^{\frac{1}{2}(v-1)} \text {. }
$$

It is easy to check that

$$
A^{-1}=\frac{1}{(k-\lambda)}\left(A^{\mathrm{T}}-\frac{\lambda}{k} S\right),
$$

where $A^{-1}$ denotes the inverse of $A$. Thus in $A=\left[a_{r s}\right]$, if $a_{r s}=1$, then the cofactor of $a_{r s}$,

$$
A_{r s}=\frac{1}{k} \operatorname{det} A
$$

Similarly for the complement $C=\left[c_{r s}\right]$, if $c_{r s}=1$, then the cofactor of $c_{r s}$, 


$$
C_{r s}=\frac{1}{v-k} \operatorname{det} C .
$$

We are assuming that $v-2 k>0$. Thus we may replace $v-2 k$ of the 1 's in the first row of $C$ by 0 's. The resulting matrix $Q$ is a 0,1 matrix satisfying

$$
|\operatorname{det} Q|=k(k-\lambda)^{\frac{1}{2}(v-1)} \text {, }
$$

but $Q$ is not an incidence matrix of a $v, k, \lambda$ configuration.

\section{REFERENCES}

1. R. H. Bruck and H. J. Ryser, The nonexistence of certain finite projective planes, Can. J. Math., 1 (1949), 88-93.

2. S. Chowla and H. J. Ryser, Combinatorial problems, Can. J. Math., $2(1950), 93-99$.

3. R. E. A. C. Paley, On orthogonal matrices, J. Math. Phys., 12 (1933), 311-320.

4. H. J. Ryser, Geometries and incidence matrices, Slaught Memorial Papers (Suppl. Amer. Math. Monthly), 62 (1955), 25-31.

5. John Williamson, Determinants whose elements are 0 and 1, Amer. Math. Monthly, 59 (1946), 427-434.

Ohio State University 